\title{
The use of Electrotactile Feedback in Cars
}

\author{
Yosuef Alotaibi \\ School of Computing Science \\ University of Glasgow \\ Glasgow, G12 8QQ, Scotland \\ UK \\ y.alotaibi.1@research.gla.ac.uk
}

\begin{abstract}
Electrotactile feedback can be used as a novel method to evoke different sensations on the skin. However, there is a lack of research exploring its use on the palm. My research focuses on investigating the use of electrotactile feedback as a mean of conveying information to car drivers with minimum distraction from the main task of driving. To achieve this, we need to manipulate its parameters such as frequency, amplitude and frequency of electrical stimulation on the palm. It also provides an understanding of the design space of electrotactile cues to be used for feedback in in-car haptics interfaces to enable designers to create effective electrotactile feedback.
\end{abstract}

Human computer interaction, In-car-haptics, Electrotactile feedback, Haptics, Electrotactile

\section{INTRODUCTION}

Car driving needs many systems to assist driving, improve safety, avoid distraction and enjoy entertainment. These systems deliver a large amount of information which comes from lots of sensors for lane departure warning, ambient vehicle detection and communication. For this, is it important to improve or implement a new system that conveys information and interact with the driver easily and intuitively.

The most common way to convey information to the driver is through the console display. This requires a visual interaction from the person driving the car, which will cause a gaze off the road and add a cognitive load and distract the driver from the primary task of driving. This leads to a huge safety risk, giving that $60 \%$ of near-crashes and incidents caused by gazing off the road Klauer et al. (2006).

Finding other means to convey notifications and warning signs to the drivers with minimum or no distraction of the road is the aim of most researchers in the field of in-car haptics. Exploring the use of senses like touch and hearing through vibrotactile and audio-tactile showed promising results in ideal driving conditions, but with limitations.

For audio-tactile, it can interfere with the in-car entertainment, or conversations. Also, in a noisy situation, it might be hard to hear it. For vibrotactile, it can be masked by the vibration of the road, making it difficult to recognise. These limitations affect driving performance, especially in times when an immediate reaction is needed. The challenge for any use of different technology is how effective it is indifferent driving conditions.

So my research focuses on conveying and communicating information to the driver while keeping the gaze on the road through the use of electrotactile feedback. The perception and acceptance of this technology were investigated through the manipulation of its parameters to find a parameter space that can be used to design the desired sensation.

\section{LITERATURE REVIEW}

\subsection{Cutaneous Stimulation}

In this research area, the focus is on the methods of conveying information to the user through the skin using tactile feedback. The use of the terms haptic and tactile can be confusing because they are used interchangeably Krol et al. (2009). The term haptic is used to describe all kinds of sensations that been evoked in the human body, including tactile/cutaneous and kinaesthetic stimulation. Tactile describes the skin's sense of mechanical, thermal, chemical and stimulations. Erp et al. (2010); Subramanian et al. (2005).

To elicit a specific feedback, it is important to understand the structure of the skin so that we can manipulate the parameters of the technology used. There are four types of mechanoreceptors in the 
human skin, and each one responsible for sensing a specific skin deformation. The four types (from the top of the skin) are Meissner corpuscles that react to low frequencies (20-70 [Hz]). After it comes Merkel cells that react to pressure, then Ruffini endings that react to shear deformation, and at the end Pacinian corpuscles that react to high frequencies (100-300[Hz]) Kajimoto et al. (2003).

\subsection{In-car haptics}

Many studies were integrating vibrotactile feedback into different parts of the car to convey information. For assisting the driver in car following, Abbink et al. (2008) supplemented Advanced Driver Assistance System (ADAS) with haptic feedback. The gas pedal was used to pass information about relative distance and velocity to the led car. This design aims to yield better situation awareness and faster response, but the analysis showed some issues. The system can be annoying and may cause fatigue. Also, there were difficulties in interpreting the correct haptic cues.

The driver's seat is another place were vibrotactile can be implemented for navigational cues. Hogema et al. (2009) used an $8^{*} 8$ matrix of vibrator motors embedded in the seat pan to communicate directional information (the four cardinal and four obliques) in an in-traffic filed study. To indicate a direction to the driver, a vibrotactile pattern of three bursts will be activated. Each lasting for $250 \mathrm{~ms}$ and are separated by $250 \mathrm{~ms}$. Directional accuracy and reaction time were measured. The results showed that the correct directional responses were high $92 \%$, and the test condition has no significant effect on the reaction time. However, some issues affected the feeling of the haptics of the seat: the driver's posture and vehicle and body vibrations.

Dass et al. (2013), Used vibrotactile in the driver's seat for lane departure warning in trucks in a driving simulator and real world. They used the backrest with the seat. Eight vibrators were used, one under each thigh in the seat, and two columns in the backrest with three vibrators each. Two designed haptic signals were compared to an auditory warning. When the vehicle's tire deviate from the visible lane marking by $8-33 \mathrm{~cm}$, a medium urgency signal will be activated. When the deviate more than $33 \mathrm{~cm}$, a high urgency signal will be activated. The findings were that the haptic and auditory signals are equally effective and that the users preferred the haptic more than the auditory.

Vibrotactile on the steering wheel for navigation was investigated by Kern et al. (2009) in a driving simulator. They placed six actuators on the steering wheel. When indicating a turn, two actuators on each side will be activated. They conducted two studies; the first one compared three types of feedback, audio, vibrotactile and a multimodal of both. The results showed that participants were correct less often, and the driving performance was decreased with vibrotactile than audio or both together. In the second study, they added visual instructions (arrows) and made different combinations of multimodal feedback. The results showed that the combination of vibrotactile and visual had a significant performance improvement.

\subsection{Electrotactile feedback}

Electrotactile feedback can elicit different sensations Djozic et al. (2015); Franceschi et al. (2015) and conveying information Kaczmarek and Haase (2003); Kajimoto et al. (2014); Kim et al. (2004)through electrical current generating a electrical filed inside the skin to stimulate the nerves. The advantages it was over mechanical tactile are a smaller and thin size, light, durable, free from mechanical resonance, and having high responsiveness Yem and Kajimoto (2017); Kajimoto (2012); Kajimoto et al. (2003). Manipulating the parameters of electrotactile is necessary to design tactile feedback. The parameters are amplitude, frequency, pulse width, type of electrical current and location of the electrodes. In the literature review below, I will go through some studies that explored the use of electrotactile feedback in different scenarios and explain the effect of each parameter in its contexts.

Kajimoto et al. (1999) developed an electrotactile display called "tactile primary colours" which is eliciting a wide range of sensations through stimulating the Meissner corpuscle (RA), Merkel cell (SAI) and Pacinian corpuscle (PC) separately. They used two methods to stimulate mechanoreceptors. They used array electrodes (eight-line electrodes), and the other method is anodic and cathodic currents. Based on the orientation of the mechanoreceptor, different method of stimulation has been used. The RA is vertical to the skin surface, while SAI and PC are horizontal. Where SAI is located in the shallower part of the skin than PC.

Based on orientation, the anodic current is used to stimulate RA, while cathodic current used to for SAI and arrayed electrodes for PC. The amplitude for the stimuli applied was $2 \mathrm{~mA}$, pulse width 200 $\mu \mathrm{S}$, and frequency of $200 \mathrm{~Hz}$. They reported that participants felt different sensations with every method of stimulations. When stimulating $\mathrm{SAI}$, at 0.2 $\mathrm{mA}$, they felt a tremble sensation. At $0.4 \mathrm{~mA}$, they felt pressure sensation. At $0.6 \mathrm{~mA}$, they felt a vibration. When stimulating RA with a frequency less than 100 $\mathrm{Hz}$, participants felt a vibration, when above $200 \mathrm{~Hz}$ it was uncomfortable. 


\section{INVESTIGATING ELECTROTACTILE FEEDBACK ON THE HAND}

\subsection{Experiment 1}

This experiment was conducted to provide an initial evaluation of electrotactile feedback and how people would react to it by stimulating their palm. This experiment aim was to answer the question, Do frequency and pulse width influence people's sense of urgency,annoyance, valence and arousal?

This was a within-participants experiment, consisted of three phases: calibration phase, training phase and experiment phase. The independent variables were frequency and pulse width. I used six equally spaced frequencies $(10 \mathrm{~Hz}, 30 \mathrm{~Hz}, 50 \mathrm{~Hz}, 70 \mathrm{~Hz}, 90$ $\mathrm{Hz}$ and $110 \mathrm{~Hz}$ ), and three values for pulse width. The first value for pulse width as a baseline value was $70 \mu \mathrm{s}$ based on a previous work done by the group using the same hardware. The second value was measured during the calibration phase as the discomfort threshold because each participant's impedance is different. The third value was the mean between the baseline value and the discomfort threshold. The combination of both produced a total of 18 stimuli. The dependent variables were the perceived urgency, annoyance, valence and arousal.

The results showed that the most significant effects in frequency were when comparing $10 \mathrm{~Hz}$ with higher frequencies. This indicates that participants couldn't tell the difference between frequencies when going above $30 \mathrm{~Hz}$. Not having a significant effect between low and high pulse width in valence indicates that the rating went down after middle pulse width. This means that the valence threshold is somewhere between middle and high. Also, the proportional relationship between urgency, annoyance and arousal as the Pulse width intensity goes up.

\subsection{Experiment 2}

This experiment would be a follow up to the first one using the same hardware and set up. The independent variables are frequency and amplitude. From the first experiment, I learned that participants couldn't tell the difference when going above 30 $\mathrm{Hz}$ so that the range would be from $5 \mathrm{~Hz}$ to $45 \mathrm{~Hz}$. The amplitude will be calibrated the same way as the pulse width. The pulse width will be kept at middle intensity for the whole experiment, giving that some participants couldn't feel most of the stimuli at low intensity. After this experiment, I will have a clear insight into the parameter space of frequency, pulse width and amplitude to design stimuli in in-car haptics.
The following changes were made compared to the first experiment. The stimulation parameters frequency and amplitude were independent variables. I used nine frequencies $(5 \mathrm{~Hz}, 10 \mathrm{~Hz}, 15 \mathrm{~Hz}, 20 \mathrm{~Hz}$, $25 \mathrm{~Hz}, 30 \mathrm{~Hz}, 35 \mathrm{~Hz}, 40 \mathrm{~Hz}, 45 \mathrm{~Hz}$ ), and three values for amplitude were defined during the calibration phase. This gave 27 stimuli from both parameters.

Having smaller steps in the range frequencies compared to the first experiment, helped us locate what frequencies have significant effect on perception. I observed that the significant effect was between the range of $5 \mathrm{~Hz}$ to $25 \mathrm{~Hz}$ and the other frequencies. At that range, Only Meissner corpuscles are activated, leading participants to distinguish between different stimuli more reliably.

In line of related work ?, the higher the level of amplitude, the higher the urgency, annoyance and arousal. I observed that amplitude has a higher impact on perception than pulse width across all levels.

\section{CONCLUSION}

The studies I conducted investigated the effects of electrotactile feedback on urgency, annoyance, valence and arousal through the manipulation of frequency, pulse width and amplitude. The aim was to explore the design space of electrotactile feedback so that we can design effective cues that might be used in a steering wheel and understand the relative importance of the different parameters. All of the parameters had a significant effect on subjective perception. Results showed perception of frequency peaked at $25-30 \mathrm{~Hz}$; above that, increases were not recognised. Frequency had generally little effect on valence but did affect the other sensations. However, there are only a few usable levels of the parameter. Increasing pulse width increased all of the perceived sensations, and gave three clear levels of the parameter. Increasing amplitude increased the ratings of urgency, annoyance and arousal, but decreased the ratings for valence, again giving three clear levels of the parameter to use. These findings give us a clearer understanding of the parameter space for designing electrotactile cues to create desired sensations. We can design messages with clearly different levels of arousal and urgency, and can see the effects on annoyance; however, valence appears harder to manipulate using electrotactile cues. this work was published in the IEEE haptics symposium Alotaibi et al. (2020). My next two experiments will be on what how many levels of each of these parameters should I use to design cues to convey information. After that, I will use electrotactile feedback on a steering wheel and measure the recognition rate. 


\section{REFERENCES}

Abbink, D. A., E. R. Boer, and M. Mulder (2008, 6). Motivation for continuous haptic gas pedal feedback to support car following. In 2008 IEEE Intelligent Vehicles Symposium, Eindhoven, Netherlands, pp. 283-290. IEEE.

Alotaibi, Y., J. H. Williamson, and S. Brewster $(2020,3)$. Investigating Electrotactile Feedback on The Hand. In 2020 IEEE Haptics Symposium (HAPTICS), Volume 2020-March, Crystal City, VA, USA, pp. 637-642. IEEE.

Dass, D. E., A. Uyttendaele, and J. Terken (2013). Haptic in-seat feedback for lane departure warning. In Proceedings of the 5th International Conference on Automotive User Interfaces and Interactive Vehicular Applications - AutomotiveUI '13, New York, New York, USA, pp. 258-261. ACM Press.

Djozic, D. J., D. Bojanic, G. Krajoski, N. Popov, and V. Ilic $(2015,11)$. Psychophysical characteristics of electrotactile stimulation: The impact of changes in stimulation pulse width and frequency on human perception. In 2015 IEEE 15th International Conference on Bioinformatics and Bioengineering (BIBE), Belgrade, Serbia, pp. 1-5. IEEE.

Erp, J. B. F. V., K.-u. Kyung, S. Kassner, J. Carter, S. A. Brewster, G. Weber, and I. Andrew (2010). Haptics: Generating and Perceiving Tangible Sensations, Volume 6192 of Lecture Notes in Computer Science. Berlin, Heidelberg: Springer Berlin Heidelberg.

Franceschi, M., L. Seminara, L. Pinna, S. Dosen, D. Farina, and M. Valle (2015). Preliminary evaluation of the tactile feedback system based on artificial skin and electrotactile stimulation. Proceedings of the Annual International Conference of the IEEE Engineering in Medicine and Biology Society, EMBS 2015-Novem, 4554-4557.

Hogema, J., S. De Vries, J. Van Erp, and R. Kiefer $(2009,10)$. A Tactile Seat for Direction Coding in Car Driving: Field Evaluation. IEEE Transactions on Haptics 2(4), 181-188.

Kaczmarek, K. A. and S. J. Haase (2003). Pattern identification and perceived stimulus quality as a function of stimulation waveform on a fingertip-scanned electrotactile display. IEEE Transactions on Neural Systems and Rehabilitation Engineering 11(1), 9-16.

Kajimoto, H. (2012, 4). Electrotactile Display with Real-Time Impedance Feedback Using Pulse Width Modulation. IEEE Transactions on Haptics 5(2), 184-188.
Kajimoto, H., N. Kawakami, A. Maeda, and S. TACHI (1999). Tactile feeling display using functional electrical stimulation. International Conference on Artificial Reality and Tele- existence.

Kajimoto, H., N. Kawakami, and S. Tachi (2003). Psychophysical evaluation of receptor selectivity in electro-tactile display. 13th Int. Sympo. on Measurement and Control in Robotics (ISMCR) (January), 3-6.

Kajimoto, H., M. Suzuki, and Y. Kanno (2014). HamsaTouch. In Proceedings of the extended abstracts of the 32nd annual ACM conference on Human factors in computing systems - CHI EA '14, New York, New York, USA, pp. 1273-1278. ACM Press.

Kern, D., P. Marshall, E. Hornecker, Y. Rogers, and A. Schmidt (2009). Enhancing Navigation Information with Tactile Output Embedded into the Steering Wheel. In Lecture Notes in Computer Science (including subseries Lecture Notes in Artificial Intelligence and Lecture Notes in Bioinformatics), Volume 5538 LNCS, pp. 42-58.

Kim, G., R. Okuno, M. Yoshida, and K. Akazawa (2004). Sensory substitution system of twochannel electrotactile stimulation for transmitting verbal information. In The 26th Annual International Conference of the IEEE Engineering in Medicine and Biology Society, Volume 4, San Francisco, CA, USA, pp. 4948-4951. IEEE.

Klauer, S., S. Klauer, T. a. Dingus, T. a. Dingus, V. L. Neale, V. L. Neale, J. Sudweeks, J. Sudweeks, D. Ramsey, and D. Ramsey (2006). The Impact of Driver Inattention On Near Crash/Crash Risk: An Analysis Using the 100-Car Naturalistic Driving Study Data. Analysis (April), 226.

Krol, L. R., D. Aliakseyeu, and S. Subramanian (2009). Haptic feedback in remote pointing. In Proceedings of the 27th international conference extended abstracts on Human factors in computing systems - CHI EA '09, New York, New York, USA, pp. 3763. ACM Press.

Subramanian, S., C. Gutwin, M. N. Sanchez, C. Power, and J. Liu (2005). Haptic and Tactile Feedback in Directed Movements. Proceedings of GOTHI05 Guidelines On Tactile and Haptic Interactions, 37-42.

Yem, V. and H. Kajimoto $(2017,1)$. Comparative Evaluation of Tactile Sensation by Electrical and Mechanical Stimulation. IEEE Transactions on Haptics 10(1), 130-134. 